\title{
Stability of massive objects in a new scalar-tensor theory
}

\author{
Gerd Schmidt, Walter Greiner, Ulrich Heinz, and Berndt Müller \\ Institut für Theoretische Physik, Johann Wolfgang Goethe Universität, 6000 Frankfurt am Main, Germany \\ (Received 2 September 1980)
}

\begin{abstract}
We define a new scalar-tensor theory with an effective gravitational coupling constant depending on a scalar field. The coupling is such that the gravitational interaction decreases with the strength of the scalar field. We show that this is not sufficient to prevent the gravitational collapse of sufficiently massive dense objects.
\end{abstract}

\section{INTRODUCTION}

The existence of black holes is closely related to the problem of singularities in space-time. In general relativity the formation of black holes is the consequence of the collapse of massive objects with masses greater than several times the solar mass. It was discussed in the literature whether in other theories of gravity, which are in accord with present gravitational experiments, the formation of event horizons or the collapse of massive objects might not occur. ${ }^{1-4}$ Good candidates for theories with the latter feature may be scalar-tensor theories, where the gravitational constant $G$ or the particle rest mass is variable (i.e., Bergmann-Wagoner theory, ${ }^{5,6}$ Nordtvedt theory, ${ }^{7}$ Brans-Dicke theory, ${ }^{8-11}$ and variable-mass theory, ${ }^{12,13}$ to mention just a few). In general, in scalar-tensor theories the metric is generated by a scalar gravitational field which acts together with nongravitational fields (matter). The scalar gravitational field itself is generated by the nongravitational fields via a wave equation in curved space-time. In this paper we discuss a new kind of scalar-tensor theory of gravity, where the gravitational constant depends on a scalar field, which itself couples to the surrounding masses via the curvature scalar.

The idea behind this model is that during the collapse of a star which in general relativity would form a black hole, in our theory a strong scalar field might be built up, which should reduce the gravitational constant until the collapse is stopped and the whole system reaches a new stable configuration. In this paper we therefore look for stable, time-independent solutions of the field equations of our theory, representing such a final configuration with very large nucleon number, surrounded by a scalar field. ${ }^{14}$ If there exist such solutions for any set of parameters, a time-dependent treatment would be necessary to find out whether a given initial configuration can reach this state. If, however, it turns out that there are no such stable end configurations if the nucleon number exceeds a definite finite boundary, this would already prove that gravitational collapse is also possible in our theory, if the initial star configuration has a mass large enough; no further time-dependent studies are then necessary. We shall show in this paper that the latter case is true. Even when varying the coupling constant between scalar and gravitational fields over the whole allowed range, the effective gravitational constant only varies within narrow bounds. It is not reduced strongly enough by the scalar field that stable solutions with arbitrary large masses are possible.

Our paper is organized as follows. In Sec. II we derive the field equations for the scalar field and the gravitational field from an action integral. In Sec. III we make a static, spherically symmetric Ansatz for the metric and derive the generalized Tolman-Oppenheimer-Volkoff equation. In Sec. IV we define a quantity which allows us to measure the stabilizing effect of the scalar field. In Sec. $V$ we make some estimates concerning the strong-coupling limit and the weak-field limit. Our numerical results are presented in Sec. VI.

\section{ACTION INTEGRAL AND FIELD EQUATIONS}

The starting point of our considerations is the conformally invariant equation for a massless scalar field ${ }^{15,16}$ :

$$
\left(\square+\frac{1}{6} R\right) \varphi=0 .
$$

In order to obtain a more general theory, we add a mass term and allow for an arbitrary coupling constant $\beta$ between scalar field $\varphi$ and curvature scalar $R$ :

$$
\left(\square+\frac{\beta}{6} R+\mu^{2}\right) \varphi=0 .
$$

This wave equation can be derived from the action integral

$$
\begin{gathered}
I^{\text {tot }}=\int\left(\frac{1}{2} g_{\mu \nu} \varphi^{\mid \mu} \varphi^{\mid \nu}-\frac{1}{2} \mu^{2} \varphi^{2}-\frac{\beta}{12} \varphi^{2} R\right. \\
+\gamma R-2 \gamma \Lambda) \sqrt{-g} d^{4} x+I^{\mathrm{ext}},
\end{gathered}
$$


where we have added the Einstein action with the cosmological term and the action of the external sources of the gravitational field $I^{\text {ext }}$ :

$$
I^{\text {ext }}=\int \mathcal{L}_{\text {matter }} \sqrt{-g} d^{4} x
$$

with the external energy-momentum tensor $T_{\mu \nu}$ :

$$
T_{\mu \nu}=\frac{2}{\sqrt{-g}}\left[\frac{\partial\left(\mathcal{L}_{\text {matter }} \sqrt{-g}\right)}{\partial g^{\mu \nu}}-\frac{\partial}{\partial x^{\sigma}} \frac{\partial\left(\mathcal{L}_{\text {matter. }} \sqrt{-g}\right)}{\left.\partial g^{\mu \nu}\right|_{\sigma}}\right] .
$$

$\gamma$ is half of the inverse gravitational constant

$$
\gamma=c^{2} / 16 \pi G
$$

and $\Lambda$ is the cosmological constant.

A first glance at the action integral (3) reveals the following possible effects of the scalar field.

(i) Instead of $\gamma$, now $\left[\gamma-(\beta / 12) \varphi^{2}\right]$ becomes the effective gravitational coupling constant. This means that the gravitational constant, as measured, e.g., by a Cavendish scale, depends on the strength of the scalar field and hence may vary as a function of space and time.

(ii) The effective mass $\left[\mu^{2}+(\beta / 6) R\right]^{1 / 2}$ of the scalar field depends on the curvature scalar $R$.

(iii) A long-range scalar field gives a contribution to the cosmological constant $\sim \mu^{2} \varphi^{2}$, so that we also get an effective cosmological constant $\Lambda_{\text {eff }}=\Lambda+\mu^{2} \varphi^{2} / 4 \gamma$. Because of its smallness ${ }^{17}$ we neglect the cosmological constant. At the same time the experimental data for $\Lambda_{\text {eff }}$ give an upper limit for the mass of the bosons.

The variation of (3) with respect to $\varphi$ and $g_{\mu \nu}$ leads to the equations for the metric and the scalar field:

$$
\begin{aligned}
&\left(\gamma-\frac{\beta}{12} \varphi^{2}\right)\left(R_{\mu \nu}-\frac{1}{2} g_{\mu \nu} R\right) \\
&=-\frac{1}{2} T_{\mu \nu}-\frac{1}{2}\left[\varphi_{\mid \mu} \varphi_{\mid \nu}-\frac{1}{2} g_{\mu \nu}\left(\varphi_{\mid \alpha} \varphi^{\mid \alpha}-\mu^{2} \varphi^{2}\right)\right] \\
&+\frac{\beta}{12}\left[\left(\varphi^{2}\right)_{\|\mu\| \nu}-g_{\mu \nu}\left(\varphi^{2}\right)^{\| \sigma_{\| \sigma}}\right] \\
& \square \varphi+\mu^{2} \varphi+\frac{\beta}{6} R \varphi=0
\end{aligned}
$$

By forming the trace of Eq. (4) one obtains an expression for the curvature scalar:

$$
R=\frac{1}{\gamma-(\beta / 12) \varphi^{2}}\left[\frac{1}{2} T_{\mu}^{\mu}-\frac{1-\beta}{2} \varphi_{\mid \mu} \varphi^{\mid \mu}+\frac{\beta}{2} \varphi \square \varphi+\mu^{2} \varphi^{2}\right] .
$$

This relation may be utilized to eliminate the curvature scalar from the Klein-Gordon equation (5):

$$
\begin{aligned}
\square \varphi+ & \frac{(\beta / 6) \varphi}{\gamma+[\beta(\beta-1) / 12] \varphi^{2}} \\
& \times\left(\frac{6}{\beta} \mu^{2} \gamma+\frac{1}{2} T_{\mu}^{\mu}+\frac{1}{2} \mu^{2} \varphi^{2}-\frac{1-\beta}{2} \varphi_{\mid \mu} \varphi^{\mid \mu}\right)=0 .
\end{aligned}
$$

\section{SPHERICALLY SYMMETRIC FIELDS AND THE GENERALIZED TOLMAN-OPPENHEIMER- VOLKOFF EQUATION}

To investigate the influence of a scalar field on the gravitational collapse, we consider the simplest system we can imagine. The investigated object and therefore the metric shall be spherically symmetric, nonrotating, and uncharged. In the following we will call such objects stars. Because we are interested in stable configurations, the metric will be required to be globally time independent. Under these assumptions, the most general form of the line element is

$$
d s^{2}=e^{\nu(r)} d t^{2}-e^{\lambda(r)} d r^{2}-r^{2} d \Omega^{2}
$$

and the scalar field $\varphi$ is a function of $r$ only.

The deflection of light in a scalar-tensor theory is less than in general relativity, since the scalar part of the gravitational field gives no contribution. (In a pure scalar theory the space-time is conformally flat and null geodesics develop as in flat space.) From solar-system experiments it is known that the contribution of a scalar field to the gravitational field must be small. We therefore make the assumption that far away from a star the scalar field does not contribute to the gravitational coupling constant. Hence the scalar field must vanish at infinity ${ }^{18}$.

$$
\varphi(r) \rightarrow 0 \text { for } r \rightarrow \infty \text {. }
$$

For the energy-momentum tensor of the stellar constituent matter we make the usual hydrodynamical Ansatz:

$$
T_{\mu}{ }^{\nu}=\operatorname{diag}(\rho(r),-p(r),-p(r),-p(r)) .
$$

In agreement with the field equations (4) and (5) the invariance of the action integral against coordinate translations gives ${ }^{19}$

$$
T^{\mu \nu} \| \nu_{\nu}=0 \text {. }
$$

If we now wish the scalar field to help stabilizing massive stars, we have to require that the effective gravitational coupling constant decreases as a function of the scalar field, i.e.,

$$
\gamma_{\text {eff }} \equiv \gamma-\frac{\beta}{12} \varphi^{2}>\gamma
$$

Hence, because $\varphi^{2}$ is positive definite, $\beta$ must be negative.

In the following we therefore take into account 
only negative values of the coupling constant $\beta$, i.e., $\beta<0$. Inserting the Ansätze (8), (9), and (10) into Eqs. (4) and (5), we get the following set of equations for the radial functions $\nu(r), \lambda(r), p(r)$, and $\varphi(r)$ :

$$
\begin{aligned}
& \nu^{\prime}=-\frac{2 p^{\prime}}{p+\rho}, \\
& e^{-\lambda}=1-2 \frac{M(r)}{r},
\end{aligned}
$$

with

$$
\begin{aligned}
& M(r) \equiv \frac{1}{4 \tilde{\gamma}_{\text {eff }}} e^{-\int \Phi} \int e^{+\int \Phi}\left[r^{2}\left(\rho+\frac{1}{2} \mu^{2} \varphi^{2}\right)-\frac{2}{3} \beta r \varphi \varphi^{\prime}\right. \\
& \left.+r^{2}\left(\frac{1}{2}-\frac{\beta}{3}\right) \varphi^{\prime 2}-\frac{\beta}{3} r^{2} \varphi \varphi^{\prime \prime}\right] d r
\end{aligned}
$$

and

$$
e^{ \pm \int \Phi} \equiv \exp \left\{ \pm \int \frac{r}{2} \frac{1}{\tilde{\gamma}_{\text {eff }}}\left[\left(\frac{1}{2}-\frac{\beta}{6}\right) \varphi^{\prime 2}-\frac{\beta}{6} \varphi \varphi^{\prime \prime}\right] d r\right\}
$$

$$
\begin{gathered}
\gamma_{\text {eff }} \text { and } \tilde{\gamma}_{\text {eff }} \text { stand for } \\
\gamma_{\text {eff }}=\gamma-\frac{\beta}{12} \varphi^{2}, \\
\tilde{\gamma}_{\text {eff }}=\gamma_{\text {eff }}-\frac{\beta}{12} \gamma \varphi \varphi^{\prime} .
\end{gathered}
$$

Furthermore, we get

$$
\begin{aligned}
& {\left[\gamma+\frac{\beta(\beta-1)}{12} \varphi^{2}\right]\left(\varphi^{\prime \prime}+\frac{2}{r} \varphi^{\prime}+\frac{\nu^{\prime}-\lambda^{\prime}}{2} \varphi^{\prime}\right) e^{-\lambda}+\frac{\beta(\beta-1)}{12} \varphi \varphi^{\prime 2} e^{-\lambda}-\gamma \mu^{2} \varphi-\frac{\beta}{12} \varphi\left(\rho-3 p+\mu^{2} \varphi^{2}\right)=0,} \\
& p^{\prime}(r)=-\frac{M(r) \rho}{r^{2}} \frac{\gamma_{\text {eff }}}{\tilde{\gamma}_{\text {eff }}}\left(1+\frac{p}{\rho}\right)\left\{\left[1+\frac{r^{3}\left(p-\frac{1}{2} \mu^{2} \varphi^{2}\right)}{4 \gamma_{\text {eff }} M(r)}\right] e^{\lambda}+\frac{r^{3}}{4 \gamma_{\text {eff }}}\left(\frac{1}{2} \varphi^{\prime 2}+\frac{2}{3} \beta r \varphi \varphi^{\prime}\right)\right\} .
\end{aligned}
$$

The last equation (17) is the generalization of the Tolman-Oppenheimer-Volkoff equation ${ }^{20-22}$

$$
p^{\prime}(r)=-\frac{M(r) \rho}{r^{2}}\left(1+\frac{p}{\rho}\right)\left[1+\frac{r^{3} p}{4 \gamma M(r)}\right] e^{\lambda}
$$

with

$$
M(r) \equiv \frac{1}{4 \gamma} \int \rho r^{2} d r
$$

Comparing (17) with $\left(17^{\prime}\right)$ we discuss the various terms: $M(r)$ is the "gravitational charge," i.e., the product of mass and gravitational constant. In the presence of the scalar field we are not able to separate mass and gravitational constant, as is apparent from Eq. (14). The term $(1+p / \rho)$ is identical in both equations and describes how gravity acts on the internal pressure. The term

$$
1+\frac{r^{3}\left(p-\frac{1}{2} \mu^{2} \varphi^{2}\right)}{4 \gamma_{\mathrm{eff}} M(r)}
$$

means that pressure generates gravity. The action of gravity upon the scale of length and time is given by the factor $e^{\lambda}$. The last factor denotes the gravitating part of the scalar field itself.

\section{GRAVITATIONAL BINDING ENERGY OF MASSIVE STARS}

Given a solution of the field equations, we can define the bare nucleon number

$$
N(R)=\int_{0}^{R} n(r) r^{2} e^{\lambda / 2} d r d \Omega
$$

where $e^{\lambda / 2} r^{2} d r d \Omega$ is the physical volume element, $n$ the particle number density, and $R$ the surface radius of the star.

The connection between $\rho$ and $n$ is given by the following relation involving the internal energy $W(n)$ :

$$
\frac{\rho}{n}=m_{N}+W(n)
$$

where $m_{N}$ is the bare mass of the constituing nucleons. In the case of incompressible matter no work is done by compression. Neglecting thermal energy $(T \simeq 0)$, therefore the internal energy is zero and the density $\rho$ is proportional to the particle number density $n$ :

$$
\frac{\rho}{m_{N}}=n=\text { const for incompressible matter } .
$$

Thus we can replace $n$ by $\rho / m_{N}$ in Eq. (18).

We now look for an expression which allows us to decide whether collapse will be generally prevented in our theory, or whether it is still the fate of very massive objects. In general relativity the criterion for gravitational collapse for stars built of incompressible matter is that for 
arbitrary but finite central pressure the mass must be smaller than the critical bound ${ }^{20}$ :

$$
M_{\mathrm{cr}}^{2}=\frac{2^{16} \pi^{2} \gamma^{3}}{3^{5} \rho}
$$

Anticipating our results we can argue as follows: In our theory the scalar field decreases with increasing central pressure, as shown in Fig. 3, becoming zero in the limit of infinite pressure. Therefore, the same criterion is applicable and we can conclude that gravitational mass of a stable object is also bounded in our theory.

We must now distinguish between limited mass and limited baryon number. In general relativity, adding a baryon to a star leads to increasing mass and decreasing radius. But, adding a baryon to a star with scalar field we cannot necessarily expect an increase in mass and decrease in radius, since the scalar field may grow and thereby reduce the effective gravitational constant, such that in effect the gravitational charge, i.e. $M$, decreases.

If we would succeed in assembling an arbitrarily large number of baryons at finite mass and finite radius, then possibly gravitational collapse need not occur. This possibility, however, is rigorously ruled out if we can show that the gain in binding energy due to the scalar field is limited. In order to establish such proof, we define the binding energy of a star according to Eq. (18):

$$
\frac{\Delta M}{M} \equiv \frac{m_{N} N(R)-M(\infty)}{M(\infty)} .
$$

We call the scalar field stabilizing if a star with scalar field contains more nucleons than a corresponding star without scalar field (i.e., in general relativity), but having the same asymptotic mass $M(\infty)$. Let $N$. be the nucleon number of a star with scalar field, $\bar{N}$ of one without. The difference between their binding energies we call $\Delta(\Delta M / M)$

$$
\begin{aligned}
\Delta\left(\frac{\Delta M}{M}\right) & \equiv \frac{m_{N} N(R)-M(\infty)}{M(\infty)}-\frac{m_{N} \bar{N}(R)-M(\infty)}{M(\infty)} \\
& =\frac{m_{N}[N(R)-\bar{N}(R)]}{M(\infty)}
\end{aligned}
$$

The greater the value of $\Delta(\Delta M / M)$, the more stabilization we have.

As long as $\Delta(\Delta M / M)$ remains bounded, gravitational collapse will not be prevented for sufficiently large baryon number, because the surface will come too close to the Schwarzschild radius to be stable. Only if we find arbitrary large values of $\Delta(\Delta M / M)$ can we hope that gravitational collapse be evaded, since an arbitrarily large number of baryons could be contained in a star with gravitational mass $M$ well below the dangerous limit.

Of course, a proper investigation of the question when exactly in this case a collapse would really occur would require a time-dependent treatment looking for the possible presence of instabilities. We now shall investigate how $\Delta(\Delta M / M)$ behaves in our theory. Since we will find that $\Delta(\Delta M / M)$ remains bounded, we will be able to conclude that gravitational collapse will not be prevented by the scalar field, except maybe in certain marginally unstable situations.

\section{APPROXIMATE SOLUTIONS}

In this section we want to give some estimates in order to understand the numerical results presented in Sec. VI. The analytical arguments presented below will not be exact, but sufficient to see the finiteness of the effective gravitational constant, to calculate its value for stars with radius near their Schwarzschild radius, and to find an expression which relates the effective gravitational constant to measurable quantities.

\section{A. Strong-coupling limit}

In the case of a large coupling constant

$$
\beta \rightarrow-\infty,
$$

Eqs. (6) and (7) simplify to

$$
\square\left(\varphi^{2}\right)=\frac{2}{-\beta} T_{\mu}^{\mu} .
$$

Because of our boundary condition (9) the mean expectation value of the scalar field over the whole space should vanish, and we can deduce from Eq. (23)

$$
\varphi^{2}=O(1 / \beta) \text {. }
$$

But if $\varphi^{2}$ is of the order $1 / \beta$, then for large $\beta$ $\left(\beta \varphi^{2}\right)$ becomes a constant:

$$
\beta \varphi^{2} \underset{|\beta| \rightarrow \infty}{\longrightarrow} \text { const . }
$$

This result is very important because it limits the inverse effective gravitational constant $\gamma_{\text {eff }}$ :

$$
\lim _{-\beta \rightarrow \infty} \gamma_{\text {eff }}=\lim _{-\beta \rightarrow \infty}\left(\gamma-\frac{\beta}{12} \varphi^{2}\right)<\infty
$$

Thus for large negative values of the coupling constant $\beta$ the stabilization (22) remains bounded [from numerical results we find $\beta \varphi^{2}=$ const for $-\beta \gtrsim 10^{5}$ (see Fig. 1)]. 


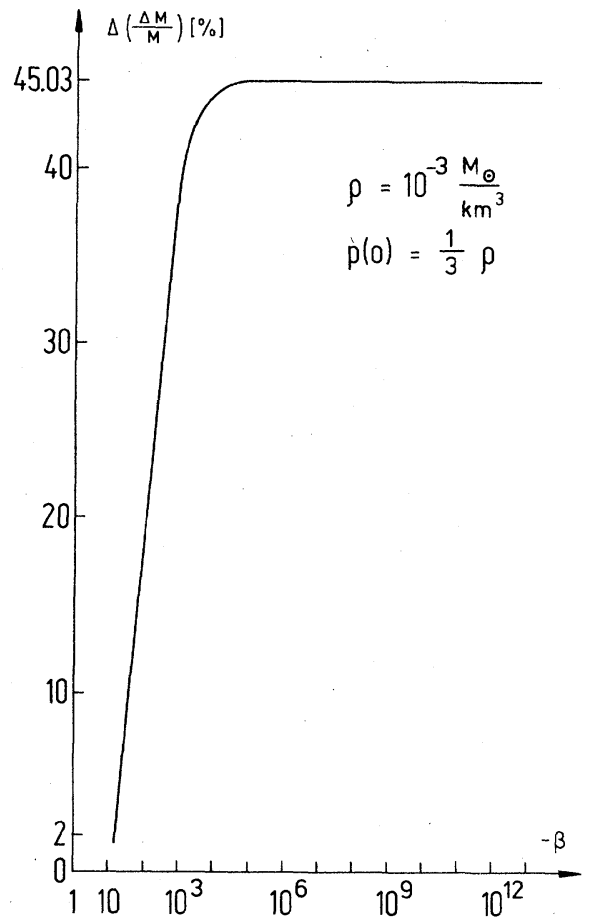

FIG. 1. The increase of binding energy with growing $(-\beta)$. For small $\beta$ the growth increases logarithmically and goes to a constant for $-\beta>10^{5}$.

\section{B. Weak-field limit}

In the case of very weak gravitational fields we can neglect $\nu^{\prime}$ and $\lambda^{\prime}$, and by setting $e^{\lambda}=1, p \ll \rho$, we obtain from (16)

$\varphi^{\prime \prime}+\frac{2}{r} \varphi^{\prime}+\frac{[\beta(\beta-1) / 12] \varphi \varphi^{\prime 2}}{\gamma+[\beta(\beta-1) / 12] \varphi^{2}}-\frac{(\beta / 12) \varphi \rho}{\gamma+[\beta(\beta-1) / 12] \varphi^{2}}=0$.

Assuming the density to be constant inside the star and taking into account the results of the previous section, we can solve Eq. (26) near the center of the star. If $\beta \varphi^{2}$ is small compared with $\gamma$ we get the solution

$$
\varphi(r)=\frac{\varphi_{0}}{r} \sin \left[\left(-\frac{\beta}{12 \gamma} \rho\right)^{1 / 2} r\right]
$$

and with $\gamma$ small compared to $\left(-\beta \varphi^{2}\right)$ we obtain

$$
\varphi(r)=\frac{\varphi_{0}}{r} \sin \left[\left(-\frac{\rho}{\beta \varphi^{2}(0)}\right)^{1 / 2} r\right]
$$

with

$$
\varphi^{2}(0)=\left(-\frac{\rho \varphi_{0}^{2}}{\beta}\right)^{1 / 2} .
$$

In the outer region, at large $r$, we make a series expansion:

$$
\begin{aligned}
& e^{\nu(r)}=1-2 \frac{M}{r}+\frac{\beta \varphi_{a}^{2}}{12 \gamma M^{2}} \frac{M^{2}}{r^{2}}+\cdots, \\
& e^{-\lambda(\nu)}=1-2 \frac{M}{r}+\frac{\left(\frac{3}{2}-\beta\right) \varphi_{a}^{2}}{6 \gamma M^{2}} \frac{M^{2}}{r^{2}}+\cdots, \\
& \varphi(r)=\frac{\varphi_{a}}{r}\left(1+\frac{M}{r}+\cdots\right) .
\end{aligned}
$$

The constants $\varphi_{0}$ in (27) and (28) and $\varphi_{a}$ in (29) are found by matching continuously the inner and the outer solution at the boundary of the star. Comparing (29) with the Eddington-Robertson expansion $^{21}$ of a metric in orders $(M / r)$ gives the following values for the measurable parameters:

$$
\begin{aligned}
& \alpha_{\mathrm{PPN}}=1, \\
& \beta_{\mathrm{PPN}}=1+\frac{\beta \varphi_{a}^{2}}{24 \gamma M^{2}}, \\
& \gamma_{\mathrm{PPN}}=1 .
\end{aligned}
$$

By measuring $\beta_{\mathrm{PPN}}$ we can set a limit on $\beta \varphi_{a}{ }^{2}$ :

$$
\frac{\beta}{12} \varphi_{a}^{2} \leqslant 2\left(\beta_{P P N}^{\exp }-1\right) M^{2} \text {. }
$$

It turns out that for stars like our Sun it is impossible to match Eq. (29) with Eqs. (27) or (28) because their radius $R$ is much larger than their gravitational radius $2 M$. However, matching would be possible for a time-dependent scalar field $\varphi(r, t)$ even if the time dependence is very small; this would require a time-dependent treatment, and we do not want to pursue this problem here. On the other hand, stars with a small ratio $2 M / R$ have high central pressures. Making the Ansatz

$$
\text { central pressure }{ }^{23} p(0)=\frac{1}{3} \rho
$$

and incompressible matter $\rho(r)=$ const, we can make a series expansion of the inner solution, which can be matched to the outer solution yielding

$$
-\frac{\beta \varphi^{2}(0)}{12}=0.15 \gamma \text {. }
$$

\section{NUMERICAL RESULTS}

We solved Eqs. (4) and (5) for stars with radius comparable with $2 M$ (say $R \sim 3 M, \ldots, 30 M$ ) by a Runge-Kutta method. The computer code was designed to find by iteration the initial value of the scalar field $(\varphi(0))$ for which the boundary condi tions are satisfied. For the equation of state we have taken that of incompressible matter ( $\rho=$ const) because one expects a maximal effect in this case, analogous to the situation in general relativity. ${ }^{21}$ The increase of binding energy with growing $|\beta|$ is shown in Fig. 1. For small $|\beta|$ it increases logarithmically and goes to a constant for $\beta<-10^{5}$. 
This is in agreement with our conclusion in Sec. $\mathrm{V}$ A that $\beta \varphi^{2}(0)$ should become constant for $(-\beta)$ going to infinity. For $\beta \varphi^{2}(0)$ we get from our numerical results

$$
-\frac{1}{12} \beta \varphi^{2}(0)=0.17 \gamma
$$

in good agreement with the estimate at the end of the previous section. Figure 2 shows an example for the scalar field inside and outside a star for a given density, pressure, and coupling constant $\beta$. The stabilization in dependence of the pressure reaches its maximum for $p(0)=\frac{1}{3} \rho$; for smaller pressure (nonrelativistic stars) and for larger pressure (radius approaching the Schwarzschild radius) the stabilization decreases. This is shown in Fig. 3. From this figure we conclude by extrapolation that for stars like our Sun the scalar field is so small that we cannot treat it numerical1y. (Indeed we found this conclusion to be true and in agreement with our results in Sec. V.) On the other hand, we see that stars just before forming a black hole have no scalar field, a result which is in agreement with statements by Hawking ${ }^{2}$ and by Bekenstein. ${ }^{1}$ As maximal stabilization effect we get

$$
\Delta\left(\frac{\Delta M}{M}\right) \cong 40 \%
$$

This means that in the best case our stars contain $40 \%$ more nucleons than stars in general relativity with the same asymptotic mass. Hence we conclude that our scalar field is not able to stabilize every star.

\section{CONCLUSIONS}

A scalar field coupled to the gravitational field in the way presented here is not able to stabilize

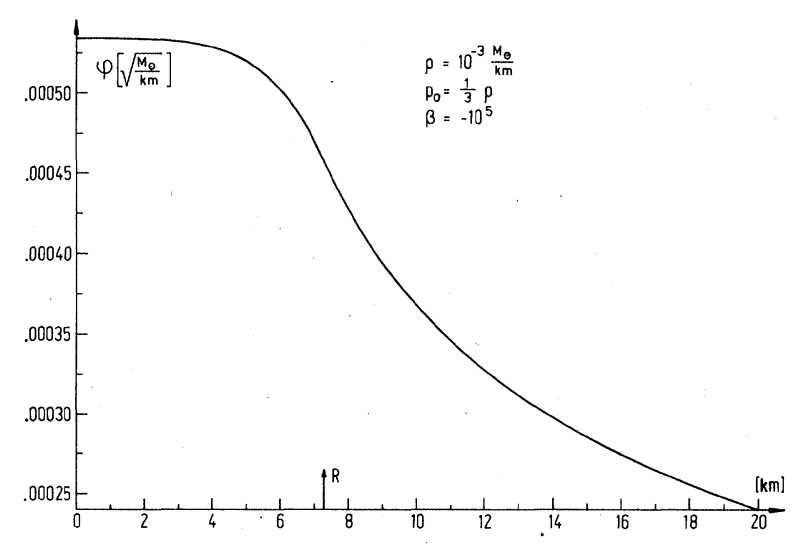

FIG. 2. The scalar field inside and outside a star of radius $R$ for a given density $\rho$, central pressure $p(0)$, and coupling constant $\beta$.

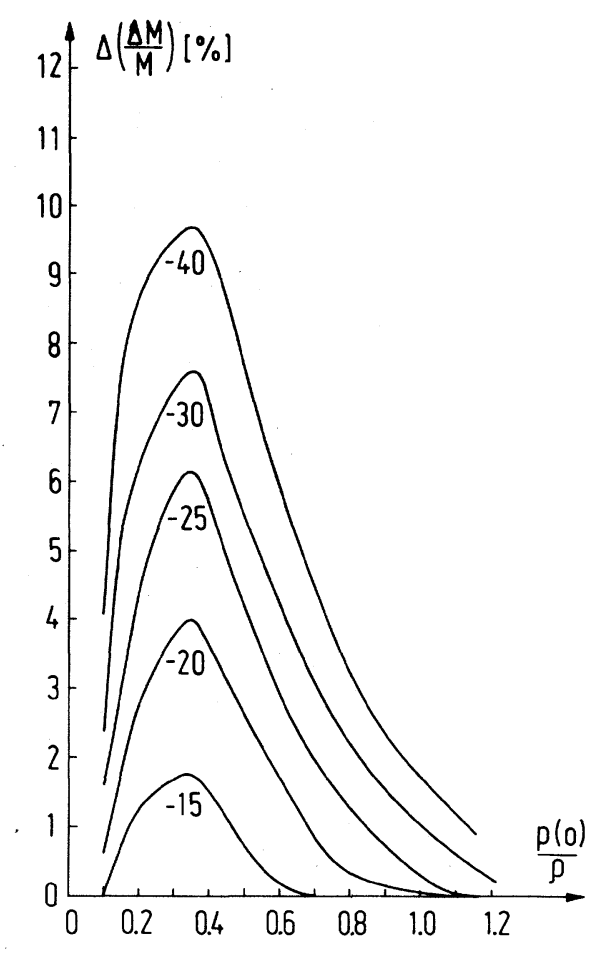

FIG. 3. The increase of binding energy in dependence of the central pressure $p(0)$ for different values of the coupling constant $\beta$. It reaches its maximum for $p(0)$ $=\frac{1}{3} \rho$, for smaller and for larger central pressure the stabilization decreases.

arbitrarily large masses against gravitational collapse. Owing to the special form of the interaction, the scalar field does not couple directly to the matter, but influences the gravitational field via the post-Newtonian parameter ${ }^{21,22} \beta_{\mathrm{PPN}}$. Present experimental data ${ }^{24}$ on the precession of the perihelion of Mercury restrict the possible range of the product $\beta \varphi^{2}(r=R)$ to values

$$
\left|\beta \varphi^{2}(R)\right| \leqslant 10^{-12} \gamma
$$

Since in the limit $\beta \rightarrow 0$ we get general relativity, we can be sure to match all experiments (today and in the future) by adapted choice of the parameter $\beta$.

We suppose that quite generally the gravitational collapse cannot be prevented by scalar fields if the action integral is of the following form:

$I^{\text {tot }}=\int\left(\gamma R-\frac{1}{2} \mu^{2} \varphi^{2}+\frac{1}{2} g^{\mu \nu} \varphi_{\mid \mu} \varphi_{1 \nu}+h(\varphi) R\right) \sqrt{-g} d^{4} x$.

This statement seems to be supported by investigations on some of these theories, but we have not been able to find a general proof of this. 


\section{ACKNOWLEDGMENTS}

We wish to thank Dr. J. Rafelski for stimulating in fruitful discussions many of the ideas presented here. This work was supported by the Deutsche Forschungsgemeinschaft (DFG) and the Gesellschaft für Schwerionenforschung (GSI).
${ }^{1}$ J. D. Bekenstein, Phys. Rev. D 5, 2403 (1972).

${ }^{2}$ S. Hawking, Commun. Math. Phys. 25, 167 (1972).

${ }^{3}$ H. J. Treder, Gravitationstheorie und Äquivalenzprinzip (Akademie, Berlin, 1971).

${ }^{4}$ J. W. Moffat, Phys. Rev. D 19, 3554 (1979).

${ }^{5}$ O. Bergmann, Int. J. Theor. Phys. 1,25 (1968).

${ }^{6}$ R. V. Wagoner, Phys. Rev. D 1, 3209 (1970).

${ }^{7}$ K. Nordtvedt, Astrophys. J. $1 \overline{61}, 1059$ (1970).

${ }^{8}$ C. Brans and R. H. Dicke, Phys. Rev. 124, 925 (1961).

${ }^{9} \mathrm{Y}$. R. Thirry, C. R. Acad. Sci. 226, $216(1948)$.

${ }^{10}$ P. Jordan, Astron. Nachr. 276, 193 (1948).

${ }^{11}$ P. Jordan, Schwerkraft und Weltall (Vieweg, Braunschweig, 1955).

${ }^{12}$ J. D. Bekenstein, Phys. Rev. D 15, 1458 (1977).

${ }^{13} \mathrm{~J}$. D. Bekenstein and A. Meisels, Phys. Rev. D $\underline{18}$, 4378 (1978).

${ }^{14}$ For the special set of parameters $\beta=+1, \mu=0$ (conformally invariant coupling) we found an analytical solution of our model which is identical to a solution found by J. D. Bekenstein, Ann. Phys. (N. Y.) 91, 75 (1975). However, since for $\beta=+1$ the effective gravitational constant is increased by the scalar field, we are not interested in that case here. Additionally, the parametrized post-Newtonian parameters of this solution are $\alpha=1, \beta=1.5$, and $\gamma=1$, which are excluded experimentally (Ref. 24) $\left(\alpha_{\text {exp }}=1, \beta_{\text {exp }}\right.$ $\left.=1.003 \pm 0.005, \gamma_{\text {exp }}=1.008 \pm 0.008\right)$ so that this solution seems not be realized in nature.
${ }^{15}$ F. Gürsey, Ann. Phys. (N. Y.) 24, 211 (1963).

${ }^{16}$ R. Penrose, Proc. R. Soc. London A284, 204 (1965).

${ }^{17}$ Ya. B. Zeldovich, Pis'ma Zh. Eksp. Teor. Fiz. 6,883 (1967) [JETP Lett. 6, 316 (1967)].

${ }^{18}$ It is also possible to have a small constant scalar field at infinity. After renormalizing the Newtonian gravitational constant and standard mass, all principal results and conclusions are the same as with no constant part except the post-Newtonian parameters $\beta$ PPN and $\gamma_{\mathrm{PPN}}$, where now a constant appears which is a measure of the contribution of the scalar field to the gravitational constant (as in Brans Dicke theory) [C. M. Will, Astrophys. J. 169, 141 (1971)]. However, according to recent experiments (Ref. 24) the relative contributions of the scalar field must be less than $1.5 \%$. ${ }^{19}$ L. D. Landau and E. M. Lifshitz, Feldtheorie (Akademie, Berlin, 1963).

${ }^{20} \mathrm{R}$. Adler, M. Bazin, and M. Schiffer, Introduction to General Relativity (McGraw-Hill, New York, 1965).

${ }^{21} \mathrm{~S}$. Weinberg, Gravitation and Cosmology (Wiley, New York, 1972).

${ }^{22} \mathrm{C}$. Misner, K. Thorne, and J. Wheeler, Gravitation (Freeman, San Francisco, 1973).

${ }^{23}$ This corresponds to an ultrarelativistic equation of state in the center of the star. Higher pressures cannot be reached by energy supply.

${ }^{24}$ I. I. Shapiro, C. C. Counselman III, and R. W. King, Phys. Rev. Lett. 36, 555 (1976). 